\title{
Papel do nutricionista em uma equipe de saúde hospitalar multiprofissional: percepção e expectativas de seus integrantes
}

\author{
Role of the nutritionist in a multiprofessional hospital health team: perception and expectations \\ of its members
}

\section{DOI: $10.37111 /$ braspenj.2020353012}

\section{Maria Janaina Ferreira de Oliveira}

Adna Jéssica Silva de Araújo²

Vanessa de Barros e Silva Mazer ${ }^{3}$

\begin{abstract}
RESUMO
Introdução: Grande parte dos indivíduos admitidos no hospital necessita de assistência nutricional, objetivando prevenir ou tratar a desnutrição, complicações infecciosas e não infecciosas decorrentes do tratamento e da doença. Nessa perspectiva, o objetivo deste estudo foi conhecer a percepção e instigar os profissionais da equipe multiprofissional de saúde das clínicas médica e cirúrgica de um hospital público regional do Estado de Pernambuco, Brasil, acerca do papel e da importância do nutricionista clínico no cuidado multiprofissional ao paciente hospitalizado. Método: Trata-se de um estudo de intervenção, baseado no modelo de pesquisa-ação, realizado com a equipe multiprofissional de saúde vinculada às clínicas médica e cirúrgica de um hospital regional de Pernambuco. Foi desenvolvido em 3 etapas: criação de um questionário dicotômico a partir de diálogo sobre a vivência dos nutricionistas clínicos do hospital; aplicação de questionário à equipe de saúde in loco; e realização de rodas de conversas baseadas na análise prévia dos questionários. Resultados: Participaram da segunda etapa 56 profissionais, dentre esses, 44 do sexo feminino e 12 do sexo masculino. A análise dos questionários aplicados à equipe multiprofissional revelou que $55,36 \%$ não sabiam da existência de nutricionista de outra área de atuação no hospital; 33,93\% nunca solicitaram parecer do nutricionista clínico, e $66,08 \%$ dos profissionais desconheciam o fluxo do Serviço de Nutrição para que a dieta chegasse ao paciente. Participaram da terceira etapa 41 profissionais, com exceção dos profissionais médicos e do terapeuta ocupacional. Conclusões: Tendo em vista que o tema alimentação e nutrição não está restrito apenas ao profissional Nutricionista e ao cenário atual de morbimortalidade e sua relação com hábitos alimentares, torna-se clara a necessidade de mais intervenções voltadas para esse tema, no intuito de que os demais profissionais conheçam ou aprimorem os seus conhecimentos em busca de um cuidado mais efetivo e humanizado.
\end{abstract}

\section{ABSTRACT}

Introduction: Most of the individuals admitted to the hospital need nutritional assistance, aiming to prevent or treat malnutrition, infectious and non-infectious complications from treatment and illness. In this perspective, the objective of this study was to know the perception, as well as to instigate the professionals of the multiprofessional health team of the medical and surgical clinics of a regional public hospital in the state of Pernambuco, Brazil, about the role and importance of the clinical nutritionist in multiprofessional care for hospitalized patients. Methods: It is an intervention study, based on the model of action-research. carried out with a multiprofessional health team linked to the medical and surgical clinics of a regional hospital in Pernambuco. It was developed in 3 stages: creation of a dichotomous questionnaire based on a dialogue about the experience of clinical nutritionists at the hospital; application of a questionnaire to the health team in loco; and conducting conversation circles based on previous analysis of the questionnaires. Results: Participated in the second stage 56 professional among these, 44 females and 12 males. The analysis of the questionnaires applied to the multiprofessional team revealed that $55,36 \%$ did not know about the existence of a nutritionist from another area of activity in the hospital; $33,93 \%$ never requested an opinion from the clinical nutritionist; and $66.08 \%$ of professionals were unaware of the flow of the Nutrition Service so that the diet reached the patient. Participated in the third stage 41 professional, with the exception of doctors and occupational therapists. Conclusions: Bearing in mind that the theme food and nutrition is not restricted only to the professional nutritionist and the current scenario of morbidity and mortality and its relationship with eating habits, it becomes clear the need for more interventions focused on this theme in order that the other professionals know or improve their knowledge in search of more effective and humanized care.

Aceito para publicação

10 de agosto de 2020

1. Nutricionista residente do Programa de Residência Multiprofissional em Atenção Hospitalar com Ênfase em Gestão do Cuidado do Hospital Regional Dom Moura (HRDM), Garanhuns, PE, Brasil.

2. Nutricionista Clínica no HRDM, Garanhuns, PE, Brasil. Mestre em Biologia Celular e Molecular Aplicada pela Universidade de Pernambuco. Especialista em Nutrição Clínica pelo Programa de Residência da Universidade de Pernambuco. Especialista em Prescrição de Fitoterápicos e Suplementação Nutricional Clínica e Esportiva pela Estácio de Sá. Tutora do Programa de Residência Multiprofissional em Atenção Hospitalar com Ênfase em Gestão do Cuidado do HRDM, Garanhuns, PE, Brasil.

3. Fisioterapeuta no HRDM, Garanhuns, PE, Brasil. Especialista em Terapia Intensiva pela Universidade Católica de Recife. Especialista e Tutora do Programa de Residência Multiprofissional em Atenção Hospitalar com Ênfase na Gestão do Cuidado do HRDM, Garanhuns, PE, Brasil. 


\section{INTRODUÇÃO}

O hospital é um ambiente que faz parte do processo de atenção à saúde, caracterizando-se como uma instituição que objetiva essencialmente a recuperação da saúde e a atenção integral ao paciente que se encontra sob a vigia dos profissionais que prestam cuidados'. Dentre esses cuidados prestados, estão os relacionados à alimentação e à nutrição, em seus diferentes graus de complexidade, considerados fundamentais para a recuperação dos pacientes'.

Para Cecílio e Merhy ${ }^{2}$, o cuidado integral ao paciente é o esforço de uma abordagem completa, holística, de cada pessoa portadora de necessidades de saúde, que, por um certo período, precise de cuidados hospitalares. A integralidade do cuidado no hospital diz respeito ao trabalho de toda a equipe, que é, por natureza, multidisciplinar.

Nesse sentido, a nutrição é uma ciência caracterizada como um conjunto de ações que compreende uma área importante na atenção oferecida aos pacientes hospitalizados, sendo definida como o estado fisiológico resultante do consumo e da utilização biológica de energia e nutrientes ${ }^{3}$. Já o ato de se alimentar é um processo cultural, indispensável à vida, que vai além das necessidades biológicas e se traduz em aspectos como a escolha, preparação e consumo de um ou vários alimentos ${ }^{3}$.

O nutricionista é um profissional generalista e/ou especialista legalmente habilitado, de acordo com a Lei Federal 8.234, de 17 de setembro de 1991, a participar de equipes multidisciplinares, criadas por entidades públicas ou particulares, e propiciar a segurança alimentar e nutricional em todas as suas áreas de atuação, sendo a alimentação e a nutrição primordiais para a promoção, a proteção e a recuperação da saúde ${ }^{1,4}$.

O Conselho Federal de Nutricionistas (CFN), por meio da Resolução de $N^{\circ}$ 600, de 25 de fevereiro de 2018, em seu Artigo $3^{\circ}$, descreve e cita as diversas áreas em que 0 nutricionista pode atuar, tais como: Nutrição em Alimentação Coletiva; Nutrição Clínica; Nutrição em Esportes e Exercício Físico; Nutrição em Saúde Coletiva; Nutrição na Cadeia de Produção, na Indústria e no Comércio de Alimentos; e a área de Nutrição no Ensino, na Pesquisa e na Extensão5.

Ademais, o CFN atribuiu aos profissionais atuantes em Nutrição Clínica a prestação de assistência nutricional e dietoterápica; promoção de educação nutricional; prestação de auditoria, consultoria e assessoria em nutrição e dietética; planejamento, coordenação, supervisão e avaliação de estudos dietéticos; prescrição de suplementos nutricionais; solicitação de exames laboratoriais, além da prestação de assistência e treinamento especializado em alimentação e nutrição a coletividades e indivíduos, sadios e enfermos, em instituições públicas e privadas, em consultório de nutrição e dietética e em domicílio ${ }^{5}$.

Apesar de o nutricionista clínico desempenhar competências específicas de sua categoria, é por meio do trabalho em conjunto com os demais profissionais que a assistência se torna mais qualificada e eficaz, mediante o desenvolvimento de um trabalho multiprofissional a partir da construção compartilhada dos diferentes saberes'.

Grande parte dos indivíduos que são admitidos no hospital necessitam de assistência nutricional, com o objetivo de prevenir ou tratar a desnutrição, complicações infecciosas e não infecciosas decorrentes de tratamento e da doença, bem como melhorar a qualidade de vida ${ }^{6}$. A triagem e a intervenção nutricional inadequadas podem resultar em agravamento do quadro clínico, bem como do estado nutricional do paciente durante a internação. Portanto, a melhor maneira para prevenire tratar agravos é o conhecimento das atribuições do nutricionista clínico por todos os profissionais da equipe multiprofissional.

Em virtude disso, o objetivo deste estudo foi conhecer a percepção e instigar os profissionais da equipe multiprofissional de saúde das clínicas médica e cirúrgica de um hospital público regional do Estado de Pernambuco, Brasil, acerca do papel e importância do nutricionista clínico no cuidado multiprofissional ao paciente hospitalizado.

\section{MÉTODO}

Trata-se de um estudo de intervenção, baseado no modelo de pesquisa-ação, no qual os atores envolvidos participam ativamente em conjunto com os pesquisadores, a fim de juntos identificarem um problema coletivo e resolverem a realidade na qual estão inseridos ${ }^{7}$.

Com o intuito de responder ao objetivo da pesquisa, realizou-se abordagem com os profissionais da equipe multiprofissional de saúde vinculada às clínicas médica e cirúrgica de um hospital da região agreste meridional de Pernambuco que presta diversos serviços especializados pelo Sistema Único de Saúde (SUS). Esse hospital está vinculado ao Programa de Residência Multiprofissional em Atenção Hospitalar com Ênfase na Gestão do Cuidado, criado em 2010, tendo como instituição formadora a Escola de Governo em Saúde Pública de Pernambuco (ESPPE) e instituída por meio da Lei n $n^{\circ} 11.129$ de $2005^{8}$.

No momento da pesquisa, o referido hospital dispunha de 85 profissionais no quadro das clínicas médica e cirúrgica, dentre eles assistentes sociais $(n=4)$, enfermeiros $(n=13)$, fisioterapeutas $(n=2)$, fonoaudiólogos $(n=2)$, médicos $(n=5)$ psicólogo $(n=1)$, técnicos de enfermagem $(n=57)$ e terapeuta ocupacional $(n=1)$. Esses profissionais foram considerados como universo da pesquisa.

Em observância às Diretrizes do Conselho Nacional de Saúde dispostas na Resolução de n 466, de 12 de dezembro de 2012, o projeto foi apresentado e aprovado pelo Comitê de Ética em Pesquisa - Autarquia Educacional de Belo Jardim (AEB) - CAAE 23702919.2.0000.5189, utilizando-se o Termo de Consentimento Livre e Esclarecido (TCLE) para os profissionais que concordaram em, livremente, participar da pesquisa?. 
Para melhor execução da pesquisa, adotaram-se como critérios de exclusão os profissionais que se encontravam de férias, atestado do serviço ou que se recusaram a assinar o TCLE.

A intervenção foi desenvolvida em 3 etapas:

- $1^{a}$ Etapa: Inicialmente, foram realizadas reuniões com todos os nutricionistas clínicos lotados no hospital $(n=6)$. No momento, foi discutida a vivência deles nas clínicas médica e cirúrgica, contemplando-se duas principais pautas, os pontos que mais os incomodavam com relação ao desconhecimento da equipe de saúde sobre o papel e importância do nutricionista clínico no hospital e como isso influencia nas intervenções diárias aos pacientes internos. Os relatos trazidos pelos nutricionistas clínicos foram registrados e serviram como base para a elaboração de um questionário dicotômico, contendo 10 questões acerca da temática (Anexo 1);
- $2^{a}$ Etapa: $O$ questionário foi autoaplicado in loco à equipe multiprofissional de saúde de ambas as clínicas, médica e cirúrgica, no período de 25 novembro a 1 dezembro de 2019, todos os dias da semana, sem quebra de continuidade, incluindo sábados, domingos e feriados, a fim de alcançar todos os profissionais plantonistas, sempre alternando entre o horário da manhã e tarde, levando em consideração a disponibilidade de tempo dos profissionais, bem como a pactuação com a gerência de ambos os setores. A finalidade do questionário foi conhecer a percepção da equipe acerca do papel e da importância do nutricionista clínico no cuidado ao paciente hospitalizado. Após a aplicação do questionário, os dados coletados foram registrados, agrupados e analisados por meio da estatística descritiva com o auxílio do programa Microsoft Excel 2016 ${ }^{\circledR}$;

Anexo 1

Questionário referente à segunda etapa do estudo

QUESTIONÁRIO

Profissão:

Tempo de formação:

Tempo de atuação no HRDM:

1. Você já teve algum contato com a disciplina de nutrição em sua formação acadêmica?

$\operatorname{Sim}(\quad) \quad$ Não ( )

2. Você conhece algo sobre a rotina e/ou papel dos Nutricionistas clínicos aqui no hospitlal?

$\operatorname{Sim}(\quad) \quad$ Não ( )

Caso queira citar algo:

3. Você sabia que existe o Nutricionista clínico e o Nutricionista da área de produção de alimentos aqui no hospital?

$\operatorname{Sim}(\quad) \quad$ Não( )

4. Durante sua atuação profissional, você já solicitou o parecer do Nutriconista clínico? Se sim, cite a(s) atuação(ôes).

$\operatorname{Sim}(\quad$ Não ( )

5. Você acha importante o trabalho do Nutricionista clínico na recuperação dos pacientes internos? $\operatorname{Sim}(\quad$ Não ( )

6. Para você a nutrição do paciente e/ou dieta que ele recebe faz a diferença em sua recuperação? $\operatorname{Sim}(\quad) \quad$ Não ( )

7. Você conhece o fluxo do serviço da nutrição para que a dieta chegue ao paciente? $\operatorname{Sim}(\quad) \quad$ Não( )

8. Você acha importante para o paciente o trabalho em conjunto com a equipe de nutrição? $\operatorname{Sim}() \quad$ Não( )

9. Você acha importante para a recuperação do paciente a comunicação entre os profissionais no serviço? $\operatorname{Sim}(\quad$ Não ( )

10. Você acha importante conhecer o real papel dos seus colegas de trabalho no serviço? $\operatorname{Sim}(\quad) \quad$ Não ( )

11. Você tem preferência de horário para realiação das rodas de conversas? Se sim, qual? $\operatorname{Sim}(\quad) \quad$ Não ( ) 
- $3^{a}$ Ełapa: Consistiu na problematização mediante rodas de conversas interdisciplinares com o públicoalvo, desenvolvidas nas salas de evolução das clínicas médica e cirúrgica, no turno da tarde, com base nos resultados da décima primeira questão, sendo realizadas todos os dias da semana, com início no dia 12 de dezembro de 2019 até o dia 18 de dezembro de 2019, a fim de atingir o maior número possível de profissionais plantonistas. A facilitação desses momentos foi feita pela nutricionista residente (também autora do projeto), uma vez que sua participação na vida cotidiana do grupo avaliado tornava possível a condução dos fatos em um contexto temporal-espacial.

Foi utilizado como instrumento de apoio um banner elaborado em conjunto com a Coordenação do Serviço de Nutrição Clínica e a nutricionista da área de Alimentação Coletiva do serviço terceirizado responsável pelo fornecimento de refeições para pacientes, acompanhantes e funcionários do hospital (Anexo 2). Esse material foi baseado na rotina do Serviço de Nutrição do hospital e na resolução de $N^{\circ} 600$, de 25 de fevereiro de 2018 do CFN.

\section{PROGRAMA DE RESIDÊNCIA MULTIPROFISSIONAL EM ATENÇÃO HOSPITALAR COM ÊNFASE EM GESTÃO DO CUIDADO DO HOSPITAL REGIONAL DOM MOURA GARANHUNS-PE TCR: “ATUAÇÃO DO NUTRICIONISTA DENTRO DE UMA EQUIPE DE SAÚDE HOSPITALAR MULTIPROFISSIONAL: ENTENDIMENTO E EXPECTATIVAS DE SEUS INTEGRANTES”}

$\begin{array}{ll}\text { * FLUXO DO SERVIÇO DE NUTRIÇÃO DO HRDM: } & \begin{array}{l}\text { Pesquisadora: Maria Janaina Ferreira de Oliveira } \\ \text { Orientadora: Adna Jessica Silva de Araújo } \\ \text { Coorientadora: Vanessa de Barros e Silva Mazer }\end{array} \\ \end{array}$

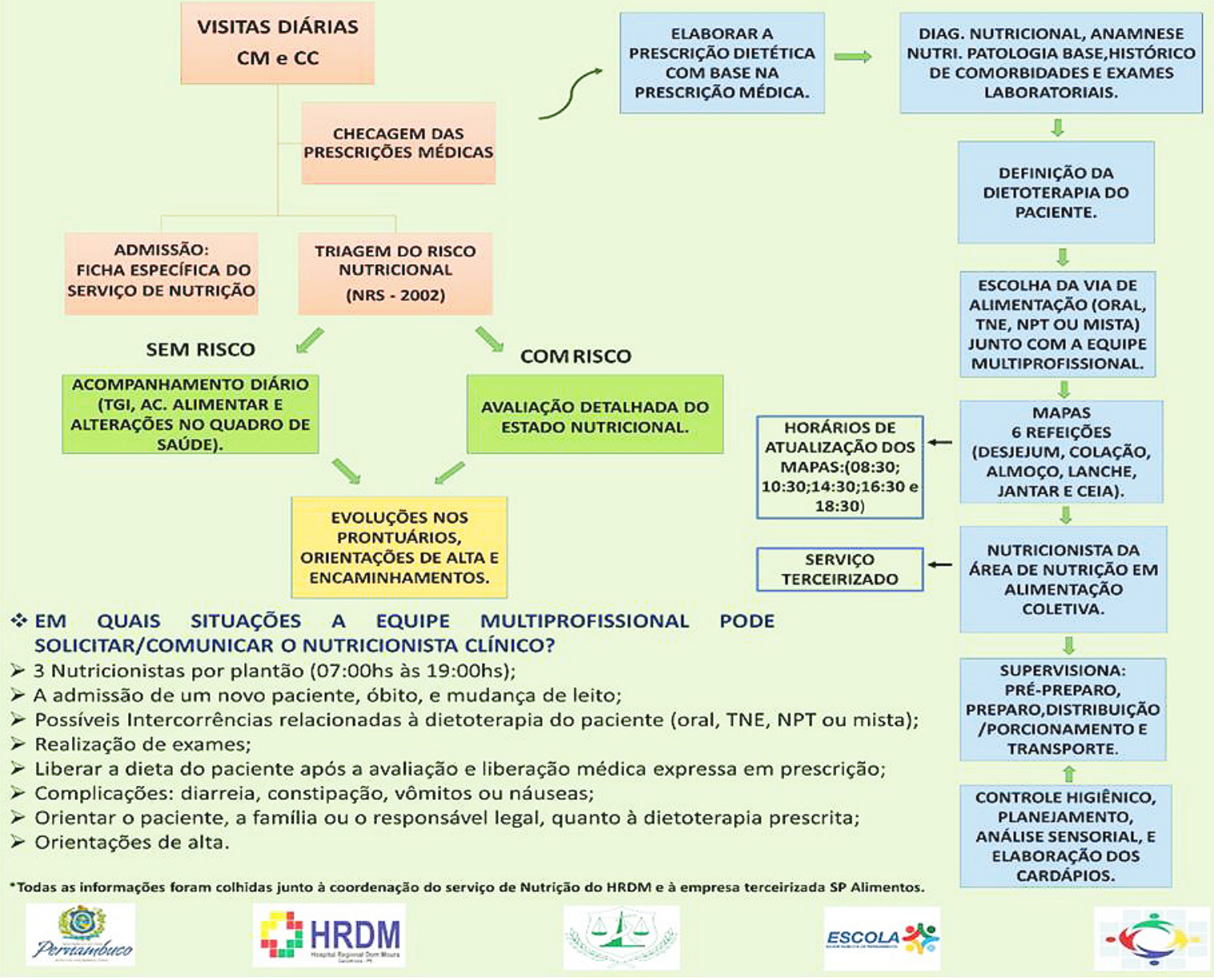




\section{RESULTADOS}

Participaram da segunda etapa do estudo 56 profissionais, correspondendo a $65,88 \%$ do total da equipe: assistentes sociais $(n=4)$, enfermeiros $(n=13)$, fisioterapeutas $(n=2)$, fonoaudiólogos $(n=2)$, médicos $(n=3)$, psicólogo $(n=1)$, técnicos de enfermagem $(n=30)$, terapeuta ocupacional $(n=1)$. Desses profissionais, 44 são do sexo feminino e 12 são do sexo masculino. O tempo médio de formação profissional foi de 17 anos para aqueles de nível médio e 15 anos para os funcionários de nível superior. Quanto ao tempo de trabalho desses profissionais no hospital, oscilou de 8 meses a 40 anos de atuação.
Os resultados relacionados à aplicação do questionário à equipe multiprofissional de saúde estão expressos nas Tabelas 1 e 2.

Os resultados apresentados na Tabela 1 evidenciam que a maioria do público-alvo $(58,92 \%)$ respondeu ter tido algum contato com a disciplina de Nutrição em sua formação acadêmica, dentre esses, enfermeiros, fonoaudiólogos, médicos e técnicos de enfermagem. Já os profissionais assistentes sociais, fisioterapeutas, psicólogos e terapeuta ocupacional referiram nunca ter tido contato com a disciplina durante sua formação.

Tabela 1 - Quantitativo das respostas obtidas por meio do questionário.

\begin{tabular}{|c|c|c|c|c|c|c|c|c|c|}
\hline \multirow[b]{2}{*}{ Questões } & \multicolumn{8}{|c|}{ Categorias profissionais } & \multirow[b]{2}{*}{$(\%)$} \\
\hline & $\begin{array}{l}\text { Assistentes } \\
\text { Sociais }\end{array}$ & $\begin{array}{l}\text { Enfer- } \\
\text { meiros }\end{array}$ & $\begin{array}{c}\text { Fisiotera- } \\
\text { peutas }\end{array}$ & $\begin{array}{c}\text { Fono- } \\
\text { audiólogos }\end{array}$ & Médicos & $\begin{array}{l}\text { Psicó- } \\
\text { logos }\end{array}$ & $\begin{array}{l}\text { Tec. de } \\
\text { Enferma- } \\
\text { gem }\end{array}$ & $\begin{array}{c}\text { Terapeuta } \\
\text { Ocupa- } \\
\text { cional }\end{array}$ & \\
\hline $\begin{array}{l}\text { 1. Você já teve algum contato com } \\
\text { a disciplina de nutrição na sua } \\
\text { formação acadêmica? }\end{array}$ & $\begin{array}{l}\text { Sim: } 0 \\
\text { Não: } 4\end{array}$ & $\begin{array}{l}\text { Sim: } 13 \\
\text { Não: } 0\end{array}$ & $\begin{array}{l}\text { Sim: } 0 \\
\text { Não: } 4\end{array}$ & $\begin{array}{l}\text { Sim: } 2 \\
\text { Não: } 0\end{array}$ & $\begin{array}{l}\text { Sim: } 1 \\
\text { Não: } 2\end{array}$ & $\begin{array}{l}\text { Sim: } 0 \\
\text { Não: } 1\end{array}$ & $\begin{array}{l}\text { Sim: } 18 \\
\text { Não: } 12\end{array}$ & $\begin{array}{l}\text { Sim: } 0 \\
\text { Não: } 1\end{array}$ & $\begin{array}{l}\text { Sim: } 58,92 \\
\text { Não: } 41,08\end{array}$ \\
\hline $\begin{array}{l}\text { 3. Você sabia que existe o nutri- } \\
\text { cionista clínico e o nutricionista } \\
\text { da área de produção de alimentos } \\
\text { aqui no hospital? }\end{array}$ & $\begin{array}{l}\text { Sim: } 1 \\
\text { Não: } 3\end{array}$ & $\begin{array}{l}\text { Sim: } 7 \\
\text { Não: } 6\end{array}$ & $\begin{array}{l}\text { Sim: } 2 \\
\text { Não: } 0\end{array}$ & $\begin{array}{l}\text { Sim: } 1 \\
\text { Não: } 1\end{array}$ & $\begin{array}{l}\text { Sim: } 0 \\
\text { Não: } 3\end{array}$ & $\begin{array}{l}\text { Sim: } 1 \\
\text { Não: } 0\end{array}$ & $\begin{array}{l}\text { Sim: } 15 \\
\text { Não: } 15\end{array}$ & $\begin{array}{l}\text { Sim: } 0 \\
\text { Não: } 1\end{array}$ & $\begin{array}{l}\text { Sim: } 44,64 \\
\text { Não: } 55,36\end{array}$ \\
\hline $\begin{array}{l}\text { 5. Você acha importante o } \\
\text { trabalho do nutricionista clínico } \\
\text { na recuperação dos pacientes } \\
\text { internos? }\end{array}$ & $\begin{array}{l}\text { Sim: } 4 \\
\text { Não: } 0\end{array}$ & $\begin{array}{l}\text { Sim: } 12 \\
\text { Não: } 1\end{array}$ & $\begin{array}{l}\text { Sim: } 1 \\
\text { Não: } 1\end{array}$ & $\begin{array}{l}\text { Sim: } 2 \\
\text { Não: } 0\end{array}$ & $\begin{array}{l}\text { Sim: } 3 \\
\text { Não: } 0\end{array}$ & $\begin{array}{l}\text { Sim: } 1 \\
\text { Não: } 0\end{array}$ & $\begin{array}{l}\text { Sim: } 30 \\
\text { Não: } 0\end{array}$ & $\begin{array}{l}\text { Sim: } 1 \\
\text { Não: } 0\end{array}$ & $\begin{array}{l}\text { Sim: } 98,21 \\
\text { Não: } 1,79\end{array}$ \\
\hline $\begin{array}{l}\text { 6. Para você, a nutrição do } \\
\text { paciente e/ou a dieta que ele } \\
\text { recebe faz a diferença em sua } \\
\text { recuperação? }\end{array}$ & $\begin{array}{l}\text { Sim: } 4 \\
\text { Não: }\end{array}$ & $\begin{array}{l}\text { Sim: } 12 \\
\text { Não: } 1\end{array}$ & $\begin{array}{l}\text { Sim: } 2 \\
\text { Não: } 0\end{array}$ & $\begin{array}{l}\text { Sim: } 2 \\
\text { Não: } 0\end{array}$ & $\begin{array}{l}\text { Sim: } 3 \\
\text { Não: } 0\end{array}$ & $\begin{array}{l}\text { Sim: } 1 \\
\text { Não: } 0\end{array}$ & $\begin{array}{l}\text { Sim: } 25 \\
\text { Não: } 5\end{array}$ & $\begin{array}{l}\text { Sim: } 1 \\
\text { Não: } 0\end{array}$ & $\begin{array}{l}\text { Sim: } 89,28 \\
\text { Não: } 10,72\end{array}$ \\
\hline $\begin{array}{l}\text { 7.Você conhece o fluxo do serviço } \\
\text { da nutrição para que a dieta che- } \\
\text { gue ao paciente? }\end{array}$ & $\begin{array}{l}\text { Sim: } 1 \\
\text { Não: } 3\end{array}$ & $\begin{array}{l}\text { Sim: } 3 \\
\text { Não: } 10\end{array}$ & $\begin{array}{l}\text { Sim: } 2 \\
\text { Não: } 0\end{array}$ & $\begin{array}{l}\text { Sim: } 1 \\
\text { Não: } 1\end{array}$ & $\begin{array}{l}\text { Sim: } 0 \\
\text { Não: } 3\end{array}$ & $\begin{array}{l}\text { Sim: } 1 \\
\text { Não: } 0\end{array}$ & $\begin{array}{l}\text { Sim: } 13 \\
\text { Não: } 17\end{array}$ & $\begin{array}{l}\text { Sim: } 0 \\
\text { Não: } 1\end{array}$ & $\begin{array}{l}\text { Sim: } 33,92 \\
\text { Não: } 66,08\end{array}$ \\
\hline $\begin{array}{l}\text { 10.Você acha importante conhecer } \\
\text { o real papel dos seus colegas de } \\
\text { trabalho no serviço? }\end{array}$ & $\begin{array}{l}\text { Sim: } 4 \\
\text { Não: } 0\end{array}$ & $\begin{array}{l}\text { Sim: } 12 \\
\text { Não: } 1\end{array}$ & $\begin{array}{l}\text { Sim: } 2 \\
\text { Não: } 0\end{array}$ & $\begin{array}{l}\text { Sim: } 2 \\
\text { Não: } 0\end{array}$ & $\begin{array}{l}\text { Sim: } 3 \\
\text { Não: } 0\end{array}$ & $\begin{array}{l}\text { Sim: } 1 \\
\text { Não: } 0\end{array}$ & $\begin{array}{l}\text { Sim: } 30 \\
\text { Não: } 0\end{array}$ & $\begin{array}{l}\text { Sim: } 1 \\
\text { Não: } 0\end{array}$ & $\begin{array}{l}\text { Sim: } 98,21 \\
\text { Não: } 1,79\end{array}$ \\
\hline
\end{tabular}


Tabela 2 - Percepções dos profissionais em relação às questões 2, 4 e 7 do questionário.

$\begin{array}{cccc}\text { Categorias } & \text { 2: Função do } & \text { 4: Solicitação de parecer do } & \text { 7: Fluxo do } \\ \text { profissionais } & \text { nutricionista clínico } & \text { nutricionista clínico } & \text { Serviço de Nutrição }\end{array}$

Assistentes Sociais

Enfermeiros

Fisioterapeutas

Fonoaudiólogos

Médicos

"Avaliação de TNE e NPT"

Psicólogo

"Avaliação nutricional do paciente, encaminhamento e orientação de alta"

Técnicos de enfermagem "Ver a dieta de um paciente" "Tipo de dieta, lembrar da dieta para o paciente" "Passar nas enfermarias visitando os pacientes"

"Supervisionar e elaborar plano alimentar dos pacientes"

"Avaliação nutricional do paciente e avaliação das necessidades e terapia nutricional"
"Em situação em que o usuário necessita de alguma alimentação específica com acesso através da rede socioassistencial"

"Chega ao serviço social a demanda de liberação de alimentação, já tentei articulação nesse sentido"

\section{"Of}

"Oferta de sonda/oral ao mesmo tempo/ volume/ duração da dieta e dietas que chegam antes do término)"

"Paciente que não aceita a dieta por via oral ou dificuldade de deglutir, alimentação enteral, avaliação nutricional e pacientes que requerem avaliação nutricional"

"Para paciente que necessita GTT e o hospital não fornecia no momento"

"Déficit nutricional"

"Solicitação quanto á questão da digestão"

\section{"Avaliação nutricional na Não souberam opinar}

"Sempre que necessário, adequar consistência e necessidades nutricionais de pacientes em atendimento fonoaudiológico em casos de disfagia e crianças em alimentação complementar" "Dieta para o paciente e discussão de necessidade de sonda"

"Já solicitei várias vezes de acordo com as condições clínicas e/ou doenças pré-existentes"

"Avaliar tipo de dieta e orientação de dieta após a alta ou para NPT"

"Paciente com queixas, perda de apetite, dúvidas sobre o problema de saúde, intolerância a certos alimentos, dificuldade em evacuar, dentre outros"

"Paciente com diarreia"

"Durante o decorrer dos plantões quando os pacientes têm dificuldade de aceitação da dieta, pedir para o nutricionista trocar"

"Para o paciente que não conseguiu deglutir"

"Avaliar a constipação do paciente"

"Paciente em dieta zero"

"Ver a dieta ou liberar"

"Necessidade de o médico prescrever o tipo" "Sei da necessidade do médico prescrever o tipo"

Não souberam opinar 
Apesar disso, observa-se que um alto percentual do público $(85,71 \%)$ demonstrou conhecer algo sobre a rotina e/ou papel dos nutricionistas clínicos no hospital, bem como puderam discorrer a respeito, como expressado na Tabela 2. Já quando perguntados sobre a existência do nutricionista da área de Alimentação Coletiva no hospital, pouco mais da metade dos profissionais $(55,36 \%)$ referiu desconhecer tal área de atuação do nutricionista.

A solicitação do parecer do nutricionista clínico foi referida por $66,07 \%$ dos profissionais avaliados, abrangendo praticamente todas as categorias da equipe multiprofissional. Tais ocasiões foram exemplificadas e apresentadas na Tabela 2.

Dos 56 profissionais participantes do estudo, 98,21\% referiram achar importante o trabalho do nutricionista clínico na recuperação dos pacientes internos, e $89,28 \%$ acreditam que a nutrição e/ou a dieta que chega até o leito do paciente faz a diferença em sua recuperação. Entretanto, mais da metade dos profissionais $(66,08 \%)$ respondeu desconhecer o fluxo do Serviço de Nutrição para que a dieta chegue até esse paciente. Os profissionais que responderam conhecer o fluxo discorreram brevemente a respeito, como demonstrado na Tabela 2.

Quando questionados sobre intervenções multidisciplinares, a maior parte dos profissionais julgou importante para a recuperação do paciente o trabalho em conjunto com a equipe de Nutrição $(94,64 \%)$, e a comunicação entre os profissionais no serviço $(98,21 \%)$, bem como o entendimento do real papel dos seus colegas de trabalho $(98,21 \%)$.

No que se refere à terceira etapa do estudo, participaram das rodas de conversas 41 profissionais, dentre esses, assistentes sociais $(n=2)$, enfermeiros $(n=12)$, fisioterapeutas $(n=2)$, fonoaudiólogos $(n=1)$, psicólogo $(n=2)$ e técnicos de enfermagem $(n=22)$, correspondendo a $73 \%$ do público participante da segunda etapa. Os médicos não participaram das rodas de conversa, tendo em vista que tais profissionais atuavam nas clínicas apenas no turno da manhã, enquanto - turno pactuado na segunda etapa da pesquisa pela maioria dos profissionais (53\%) foi o da tarde. A profissional de terapia ocupacional também não participou, visto estar ausente nos momentos de realização.

Nas rodas de conversas, foram apresentadas e discutidas as seguintes pautas: diferenças e atribuições do nutricionista clínico e do nutricionista da área de Alimentação Coletiva, situações em que a equipe multiprofissional de saúde pode solicitar o parecer do nutricionista clínico, bem como o fluxo e a rotina do Serviço de Nutrição para que a dieta chegue ao paciente. Essas pautas foram obtidas a partir da análise das respostas contidas nos questionários aplicados na segunda etapa, utilizando como base as questões 3 , 4 e 7, que apresentaram uma maior frequência de "não" como resposta.

\section{DISCUSSÃO}

No Brasil, a transição do perfil nutricional passou da desnutrição para a obesidade, exigindo a necessidade de mudanças nos serviços de saúde para dar suporte a essa nova demanda $^{10}$. Além disso, vem ocorrendo também a transição epidemiológica no perfil de morbimortalidade, que mostra as doenças crônicas não transmissíveis (DCNT) representando cerca de $75 \%$ das causas de morte na população ${ }^{11}$. A Organização Mundial de Saúde (OMS) 12 aponta que os hábitos alimentares inadequados da população consistem no principal fator de risco para a alta carga de doenças, principalmente as DCNT.

Seguindo nesse contexto, Souza ${ }^{13}$ retifica que, das dez principais causas de morte entre os brasileiros, seis estão relacionadas à alimentação: doenças cardíacas, doenças cerebrovasculares, demências, doenças renais crônicas, diabetes e problemas hepáticos crônicos. Já que a relação entre nutrição e saúde é tão clara, seria válido considerar que todos os profissionais atuantes na área hospitalar tivessem um conhecimento adequado sobre o assunto. Entretanto, o resultado do presente estudo demonstra um cenário diferente, em que praticamente metade dos profissionais entrevistados referiram não ter tido contato com nenhuma disciplina de Nutrição em sua formação.

Em um estudo semelhante, perguntou-se aos profissionais de uma equipe de saúde sobre seus conhecimentos em Nutrição. Os resultados obtidos mostraram que 60\% dos entrevistados responderam que já tiveram, durante sua formação acadêmica a disciplina de Nutrição, 27\% relataram ter assistido à disciplina em um dos semestres de sua graduação, e 13\% não tiveram contato com a disciplina em sua formação acadêmica ${ }^{14}$. Essa lacuna enaltece a importância da realização de pós-graduações ou cursos de atualização voltados para a educação em serviço, destinados às categorias profissionais que integram a área de saúde. Isso favoreceria a visão diferenciada da atenção e do cuidado, na qual não apenas as diferentes competências profissionais são reconhecidas, mas devem efetivamente estar integradas. Observou-se que a grande maioria dos profissionais do presente estudo mostrou conhecer algo sobre a rotina e/ ou papel dos nutricionistas clínicos do hospital. Os pontos mais citados como parte da rotina do nutricionista clínico, de acordo com a Tabela 1, foram referentes à avaliação nutricional do paciente e vias de alimentação (oral, TNE e NPT e liberação de dietas).

Nessa lógica, no estudo de Pereira e Oliveira ${ }^{14}$, realizado com integrantes de equipes multidisciplinares de hospitais, tendo sido os profissionais de saúde também entrevistados sobre a rotina do nutricionista no ambiente hospitalar, identificou-se que $37 \%$ dos entrevistados relataram desconhecer algo sobre, 30\% reportaram conhecer, 
porém não citaram, e 33\% mencionaram ter tido conhecimento em algum momento.

Saar e Trevizan ${ }^{15}$ analisaram a visão de uma equipe de saúde sobre os papéis de seus integrantes e evidenciaram que os demais profissionais da equipe veem o nutricionista na unidade hospitalar como um profissional responsável por prestar auxílio na dieta, educação alimentar e avaliação nutricional dos pacientes. Ainda, o nutricionista foi visto pela equipe como o único profissional capaz de elaborar cardápios adequados às necessidades individuais dos internos ${ }^{15}$.

No presente estudo, algumas categorias profissionais citaram a necessidade da prescrição médica para posterior conduta do nutricionista, tal como Saar e Trevizan ${ }^{15} \mathrm{em} \mathrm{seu}$ estudo, demonstrando que o nutricionista foi percebido pela equipe multiprofissional como um profissional dependente da categoria médica para desenvolver suas ações assistenciais, bem como consideram o nutricionista como pouco participativo, mesmo sendo considerado um profissional importante para a equipe no cuidado ao paciente.

As falas em ambos os estudos demonstraram que ainda existem resquícios nas equipes de saúde do modelo médicoassistencial, exercido de modo que o médico é colocado hierarquicamente acima de todas as demais categorias. No entanto, esse paradigma vem se rompendo desde a criação e implantação do SUS por meio da Lei de $n^{\circ} 8.080$, de 19 de setembro de 1990, que traz o princípio doutrinário da integralidade da assistência, enfatizando a importância do cuidado prestado de forma multiprofissional e interdisciplinar como estratégia na busca de uma assistência à saúde integral, efetiva e com qualidade ${ }^{16,17}$.

Apesar da desnutrição não ser reconhecida por parte de algumas categorias profissionais que prestam 0 cuidado ao paciente ${ }^{18}$, observou-se, no presente estudo, que muitos profissionais já solicitaram o parecer do nutricionista clínico em algum momento de sua atuação, evidenciando, assim, que esses profissionais reconhecem de alguma forma o fazer do nutricionista clínico no cuidado ao paciente.

Ao longo do estudo, foram discutidas questões sobre a importância da comunicação e do trabalho em conjunto entre os profissionais no serviço hospitalar. Nessa lógica, Peruzzo et al. ${ }^{19}$ reforçam que é necessária a colaboração, troca e complementaridade para que aconteça de fato o trabalho em equipe, bem como o diálogo e as relações horizontais, vistas como fundamentais na realização do cuidado integral e melhorias na qualidade do serviço prestado aos pacientes.

No entanto, alguns aspectos podem interferir no desenvolvimento da integralidade em saúde, como: profissionais não solidários, assistência em saúde hierarquizada voltada para a medicina curativa, incapacidade de criação de vínculos dos profissionais no serviço devido à alta rotatividade, diferenças sociais entre os membros da equipe, quantitativo de profissionais insuficientes, assim como a mão de obra não qualificada $^{20}$.

Assim, para que as diferentes categorias profissionais possam dispor de colaboração recíproca equilibrada, é necessário que se tenha comunicação e empatia, abarcando os diferentes saberes em cada um de seus membros. Essas práticas levam, involuntariamente, ao desenvolvimento do cuidado integral em saúde ${ }^{21}$.

Além disso, é primordial que os profissionais da equipe compreendam o papel de seus colegas no serviço. Esse tipo de atitude possibilita, ainda, a construção de uma visão global das diferentes terapêuticas e de cada situação que acontece no serviço, contribuindo para alcançar um melhor prognóstico dos pacientes, uma vez que o hospital é um ambiente complexo onde acontecem várias intervenções multiprofissionais, e a harmonia se faz fundamental na construção desse cenário ${ }^{22,23}$.

É importante destacar que o presente estudo apresenta algumas limitações. A primeira diz respeito ao curto período destinado para a execução do estudo, impossibilitando a realização da avaliação da intervenção. Assim, sugere-se que outros nutricionistas possam desenvolver trabalhos que possibilitem avaliar a intervenção, bem como dar continuidade ao proposto nesse estudo.

Uma outra limitação foi a dificuldade de incluir todos os profissionais das diferentes categorias vinculadas às clínicas em todas as etapas do estudo, pois o horário das rodas de conversas foi pactuado, limitando, assim, a participação dos profissionais que discordaram do horário proposto pela maioria, bem como as permutas entre as duas clínicas realizadas pelos profissionais técnicos de enfermagem, limitando o quantitativo total desses profissionais no estudo. Por último, a escassez de literatura sobre a temática também limitou a discussão dos resultados aqui obtidos, revelando, assim, a necessidade de mais publicações científicas, bem como maior valorização do tema.

Nessa perspectiva, na busca pelo cuidado integral em saúde, o hospital onde foi realizado o presente estudo conta com a potencialidade de ter a Residência Multiprofissional em Saúde, onde os profissionais trabalham em grupos, inseridos nas equipes de saúde vinculadas às clinicas, rompendo com os paradigmas presentes nas relações entre os profissionais de saúde no serviço. No entanto, mesmo tendo a Residência, muitos dos profissionais ainda têm a dificuldade de saber a função e o fluxo do trabalho do nutricionista no hospital, valendo, portanto, a sugestão de que as gerências dos setores hospitalares e os programas de residência estejam atentos a essas situações. 


\section{CONCLUSÃO}

Considera-se que, a partir da intervenção desenvolvida, foi possível inserir a temática do estudo no serviço, conhecer a percepção da equipe multiprofissional de saúde, bem como instigá-los sobre a importância do nutricionista clínico no cuidado integral ao paciente hospitalizado. Notou-se que muitos profissionais da equipe se surpreenderam com a temática proposta. No entanto, mostraram-se interessados e abertos a participar de todas as etapas do estudo.

Tendo em vista que o tema alimentação e nutrição não está restrito apenas ao profissional de Nutrição e levando em consideração o cenário atual de morbimortalidade e sua relação com hábitos alimentares, torna-se clara a necessidade de mais intervenções voltadas para esse tema, no intuito de que outras categorias profissionais conheçam ou aprimorem os seus conhecimentos em busca de um cuidado mais ampliado e humanizado.

Retifica-se aqui a importância do nutricionista clínico não apenas como um membro da equipe multiprofissional de saúde, mas como um profissional essencial e capacitado no cuidado integral aos pacientes, cabendo a ele garantir o aporte de nutrientes necessários para a recuperação do estado nutricional do paciente, bem como todas as operações inerentes à assistência nutricional.

\section{REFERÊNCIAS}

1. Gonçalves NEXM. O nutricionista que atua em serviços hospitalares de nutrição: competências profissionais e estratégias gerenciais [dissertação de mestrado]. Ribeirão Preto: Universidade de São Paulo, Escola de Enfermagem de Ribeirão Preto; 2016.

2. Cecílio L, Merhy EE. Integralidade do cuidado como eixo da gestão hospitalar. Construção da integralidade, cotidiano, saberes e práticas em saúde. Rio de Janeiro: Abrasco; 2003.

3. Mezzomo IB. Os serviços de alimentação: planejamento e administração. $6^{\mathrm{a}}$ ed. São Paulo: Manole; 2015.

4. Brasil. Lei n. 8.234, de 17 de Setembro de 1991. Regulamenta a profissão de Nutricionista e determina outras providências. Brasília Diário Oficial da União; 1991. [cited 2020 Mar 11]. Available from: https:/www.camara.leg.br/proposicoesWeb/ prop_mostrarintegra;jsessionid=A0E1E04CB765432355C415 327108F653.proposicoesWebExterno2? codteor $=665690 \&$ filen ame=LegislacaoCitada+-PL+5439/2009

5. Brasil. Conselho Federal de Nutricionista - CFN. Resolução no 600 , de 25 de fevereiro de 2018 que dispõe sobre definição das áreas de atuação do nutricionista e suas atribuições, estabelecem parâmetros numéricos de referência, por área de atuação e dá outras providências. Brasília: Diário Oficial da União; 2018. [cited 2020 Mar 10]. Available from: https://www.cfn.org.br/ wp-content/uploads/resolucoes/Res_600_2018.htm

6. Zanin AFF, Lima RM, Lima CAF, Älbertini SM, Lamari NM. Relevância do nutricionista na diminuição de reinternações hospitalares. Arq Ciênc Saúde. 2017;24(2):51-9.
7. Costa EP, Politano PR, Pereira NA. Exemplo de aplicação do método de Pesquisa-ação para a solução de um problema de sistema de informação em uma empresa produtora de cana-de-açúcar. Gest Prod. 2014;21(4):895-905.

8. Brasil. Lei no 11.129, de 30 de junho de 2005. Institui o Programa Nacional de Inclusão de Jovens - Pro Jovem; cria o Conselho Nacional da Juventude - CNJ e a Secretaria Nacional de Juventude; altera as Leis n. 10.683, de 28 de maio de 2003, e 10.429, de 24 de abril de 2002; e dá outras providências. Brasília: Diário Oficial da União; 2005. [cited 2020 Mar 11]. Available from: www.planalto. gov.br/ccivil_03/_Ato2004-2006/2005/Lei/L11129.htm

9. Brasil. Resolução no 466, de 12 de dezembro de 2012. Dispõe sobre diretrizes e normas regulamentadoras de pesquisas envolvendo seres humanos. Diário Oficial da República Federativa do Brasil. Brasília: Diário Oficial da União; 2013. [cited 2020 Mar 10]. Available from: https://conselho.saude.gov.br/resolucoes/2012/Reso466.pdf

10. Haack A, Fortes R, Ali BA, Alvarenga AP. Políticas e programas de nutrição no Brasil da década de 30 até 2018: uma revisão da literatura. Com Ciências Saúde. 2018;29(2):126-38.

11. Malta DC, Iser BPM, Chueiri PS, Stopa SR, Szwarcwald CL, Schmidt MI, et al. Cuidados em saúde entre portadores de diabetes mellitus autorreferido no Brasil, Pesquisa Nacional de Saúde, 2013. Rev Bras Epidemiol. 2015;18(Supl. 2):17-32.

12. Organização Mundial de Saúde(OMS). Plano de ação global para prevenção e controle de doenças não transmissíveis 2013-2020. Geneva: OMS; 2013. [cited 2020 Mar 20]. Available from: http:// www.who.int/nmh/events/ncd_action_plan/em/

13. Souza EB. Transição nutricional no Brasil: análise dos principais fatores. Cad UniFOA. 2010;5(13):49-53.

14. Pereira JO, Oliveira EF. A importância do profissional nutricionista no âmbito hospitalar: CIEGESI - Conferência Internacional de Estratégia em Gestão, Educação e Sistemas de Informação. Goiânia-GO; 2012.

15. Saar SRC, Trevizan MA. Papéis profissionais de uma equipe de saúde: visão de seus integrantes. Rev Latino-Am Enfermagem. 2007;15(1):106-12.

16. Brasil. Lei no 8.080 de 19 de setembro de 1990. Lei Orgânica da Saúde. Dispõe sobre as condições para a promoção, proteção e recuperação da saúde, a organização e o funcionamento dos serviços correspondentes e dá outras providências. Brasília: Diário Oficial da União; 1990. [cited 2020 Mar 11]. Available from: http://www.planalto.gov.br/ccivil_03/leis/18080.htm

17. Silva MVS, Miranda GBN, Andrade MA. Sentidos atribuídos à integralidade: entre o que é preconizado e vivido na equipe multidisciplinar. Interface. 2017;21(62):589-99.

18. Silva FR, Bezerra CC, Stanich P, Scorza CS, Batista REA. Triagem nutricional de pacientes internados no serviço de emergência. BRASPEN J. 2017;32(4):353-61.

19. Peruzzo HE, Bega AG, Lopes APAT, Haddad MCFL, Peres AM, Marcon SS, et al. Os desafios de se trabalhar em equipe na estratégia saúde da família. Esc Anna Nery. 2019; 22(4):e20170372.

20. Uchôa AC, Vieira RMV, Rocha PM, Rocha NSD, Maroto RM. Trabalho em equipe no contexto da reabilitação infantil. Physis Rev Saúde Coletiva. 2012;22(1):385-400.

21. Duarte MLC, Boeck JN. O trabalho em equipe na enfermagem e os limites e possibilidades da estratégia saúde da família. Trab Educ Saúde. 2015;13(3):709-20.

22. Queiroz E, Araújo TCCF. Trabalho de equipe em reabilitação: um estudo sobre a percepção individual e grupal dos profissionais de saúde. Rev Paidéia. 2009;19(43):177-87.

23. Camelo SHH. O trabalho em equipe na instituição hospitalar: uma revisão integrativa. Cogitare Enferm. 2011;16(4):734-40.

Local de realização do estudo: Hospital Regional Dom Moura, Garanhuns, PE, Brasil.

Conflito de interesse: Os autores declaram não haver. 